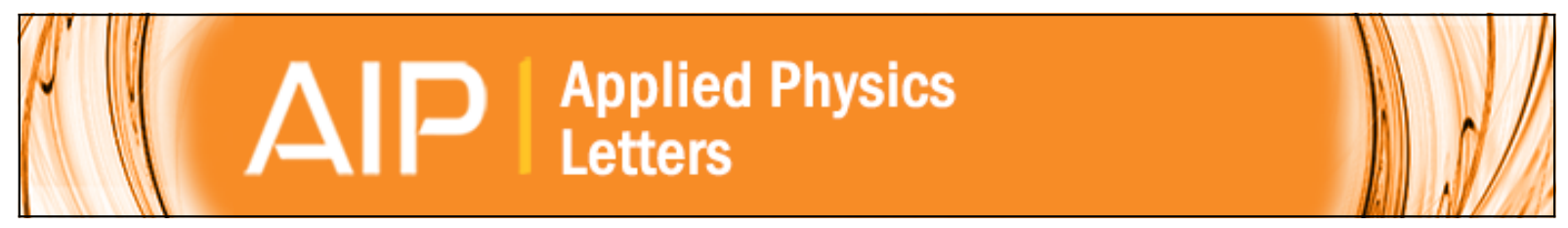

\title{
Light emission efficiency and dynamics in silicon-rich silicon nitride films
}

L. Dal Negro, J. H. Yi, J. Michel, L. C. Kimerling, T.-W. F. Chang, V. Sukhovatkin, and E. H. Sargent

Citation: Applied Physics Letters 88, 233109 (2006); doi: 10.1063/1.2208378

View online: http://dx.doi.org/10.1063/1.2208378

View Table of Contents: http://scitation.aip.org/content/aip/journal/apl/88/23?ver=pdfcov

Published by the AIP Publishing

\section{Articles you may be interested in}

Nonlinear optical properties of low temperature annealed silicon-rich oxide and silicon-rich nitride materials for silicon photonics

Appl. Phys. Lett. 100, 021109 (2012); 10.1063/1.3675882

Thermal annealing effects on photoluminescence properties of carbon-doped silicon-rich oxide thin films implanted with erbium

J. Appl. Phys. 109, 093521 (2011); 10.1063/1.3582090

Silicon nanocluster-sensitized emission from erbium: The role of stress in the formation of silicon nanoclusters

J. Appl. Phys. 104, 123108 (2008); 10.1063/1.3050324

Light emission from silicon-rich nitride nanostructures

Appl. Phys. Lett. 88, 183103 (2006); 10.1063/1.2191956

The thermo-optic effect of Si nanocrystals in silicon-rich silicon oxidethin films

Appl. Phys. Lett. 85, 2526 (2004); 10.1063/1.1798395

\section{AIP $\left.\right|_{\text {APL Photonics }}$}

APL Photonics is pleased to announce Benjamin Eggleton as its Editor-in-Chief

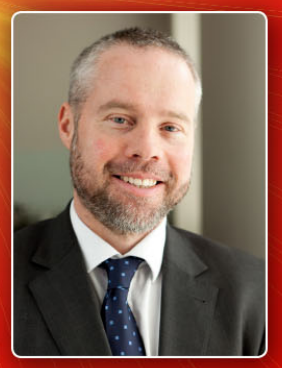




\title{
Light emission efficiency and dynamics in silicon-rich silicon nitride films
}

\author{
L. Dal Negro, ${ }^{\text {a) }}$ J. H. Yi, J. Michel, and L. C. Kimerling \\ Massachusetts Institute of Technology, 77 Massachusetts Avenue, Cambridge, Massachusetts 02139 \\ T.-W. F. Chang, V. Sukhovatkin, and E. H. Sargent \\ University of Toronto, 10 King's College Road, Toronto, Ontario, M5S 3G4, Canada
}

(Received 16 December 2005; accepted 17 April 2006; published online 7 June 2006)

\begin{abstract}
Light-emitting Si-rich silicon nitride (SRN) films were fabricated by plasma enhanced chemical vapor deposition followed by thermal annealing and the SRN external quantum efficiency was measured. The SRN light emission temperature dependence and recombination dynamics were also studied. Small emission thermal quenching from 4 to $330 \mathrm{~K}$ with wavelength dependent, nanosecond recombination lifetime was observed. Light emission from SRN systems can provide alternative routes towards the fabrication of efficient Si-based optical devices. (C) 2006 American Institute of Physics. [DOI: 10.1063/1.2208378]
\end{abstract}

Photon emission from silicon originates from lowprobability phonon-mediated transitions that compete unfavorably with fast nonradiative deexcitation paths, such as Auger and free carrier recombinations.

In view of these limitations, several strategies have been recently developed to engineer Si into a more efficient lightemitting material. ${ }^{1-4}$ Quantum confinement has led to a dramatic improvement of the light generation efficiency in $\mathrm{Si}$ nanostructures. $^{4-7}$ High emission efficiencies have been achieved in oxidized porous $\mathrm{Si}^{5-7}$ and sizable optical gain from $\mathrm{Si}$ nanocrystals ( $\mathrm{Si}$-ncs) in a Si-rich silicon oxide (SRO) film has been recently demonstrated by several groups. ${ }^{4-8-12}$ The presence of $\mathrm{Si}=\mathrm{O}$ double bonds ${ }^{13-15}$ at the surface of small Si-nc is believed to have a dramatic impact on the light emission properties of these systems. However, porous $\mathrm{Si}$ and SRO-based systems are not suitable for the fabrication of stable and efficient electroluminescent devices due to the difficult electron injection in an oxide-based matrix.

It is therefore necessary to investigate alternative complementary metal oxide semiconductor (CMOS)compatible approaches that can lead to intense light emission from nanostructured silicon systems that are also compatible with efficient and stable electrical excitation. An intriguing possibility is offered by the nucleation of Si clusters in dielectric hosts with smaller band gaps than $\mathrm{SiO}_{2}$. Visible and near-infrared light-emitting Si-ncs embedded in silicon nitride matrices have been recently demonstrated ${ }^{16,17}$ and visible electroluminescence has been reported. ${ }^{18}$

In this letter we report on a comparison of the formation parameters and external quantum efficiencies of Si-rich silicon nitride (SRN) films obtained by plasma enhanced chemical vapor deposition (PECVD) and (SRO) films deposited by magnetron sputtering. In addition, by studying the temperature dependent light emission and recombination dynamics of SRN films we demonstrate that quantum confinement cannot explain the optical properties of our SRN samples.

The SRN samples were fabricated by PECVD and the SRO samples were fabricated by reactive magnetron sputtering deposition as described in detail elsewhere. ${ }^{17,19}$ All the

\footnotetext{
${ }^{a}$ Present address: Department of Electrical and Computer Engineering, Bos-
} ton University, Boston, MA 02215; electronic mail: dalnegro@bu.edu samples have comparable thicknesses $(\approx 700 \mathrm{~nm})$ and have been deposited on transparent fused silica substrates and annealed at different temperatures ranging from 500 to $1250{ }^{\circ} \mathrm{C}$. Room temperature photoluminescence (PL) experiments were performed using a $488 \mathrm{~nm}$ Ar-ion pump laser and a single grating monochromator coupled to a nitrogen cooled InGaAs phototube. External photoluminescence quantum efficiency (PLQE) measurements were performed using a calibrated integrating sphere setup as described in Ref. 20. Photoluminescence time decay measurements were performed using the $400 \mathrm{~nm}$ second harmonic line of a Ti:sapphire laser (pulse width $\sim 100 \mathrm{fs}$, repetition rate $=25 \mathrm{MHz}$, wavelength tuned to $800 \mathrm{~nm}$ ) and a timecorrelated single photon counting system with a time resolution of $50 \mathrm{ps}$.

In Fig. 1(a1) we show the trends of the integrated PL intensity versus the SRN film refractive index (measured at $1.3 \mu \mathrm{m}$ by prism coupling technique) and postdeposition annealing temperature [Fig. 1(a2)]. We found that the best light emission performances of SRN films were obtained for samples with the highest $\mathrm{Si}$ content (a refractive index of approximately 2.24) after thermal annealing at $700{ }^{\circ} \mathrm{C}$ for 10 min. Figures 1(b1) and 1(b2) show the light emission optimization trends versus the film refractive index and the annealing temperature $(1 \mathrm{~h})$ for SRO films deposited by magnetron sputtering. The corresponding SRO film stoichiometry varies between 33 and 53 at. $\% \mathrm{Si}$, as measured by Rutherford backscattering analysis. ${ }^{19}$ In the SRO case we found that the integrated PL intensity is maximized for films with a refractive index of $1.7(\sim 38$ at. $\% \mathrm{Si})$ and annealed at $1150{ }^{\circ} \mathrm{C}$ for $1 \mathrm{~h}$. These trends result from a competitive interplay between $\mathrm{Si}$ cluster size, emission efficiencies, and $\mathrm{Si}$ cluster density. Our data indicate that a major difference in the formation kinetics exists between SRN and SRO lightemitting systems. The optimum annealing time $(1 \mathrm{~h})$ and temperature $\left(1150^{\circ} \mathrm{C}\right)$ required to maximize the $\mathrm{Si}$-nc light emission in oxide systems are much larger than what are required for $\mathrm{Si}-\mathrm{nc}$ embedded in $\mathrm{Si}$ nitride hosts. This suggests that the Si-nc growth kinetics in an amorphous nitride environment results in the formation of small Si clusters at a faster rate and at a lower annealing temperature than in the SRO case. Indeed, small size (1-2 nm) Si-ncs are formed in the amorphous nitride matrix as confirmed independently by 

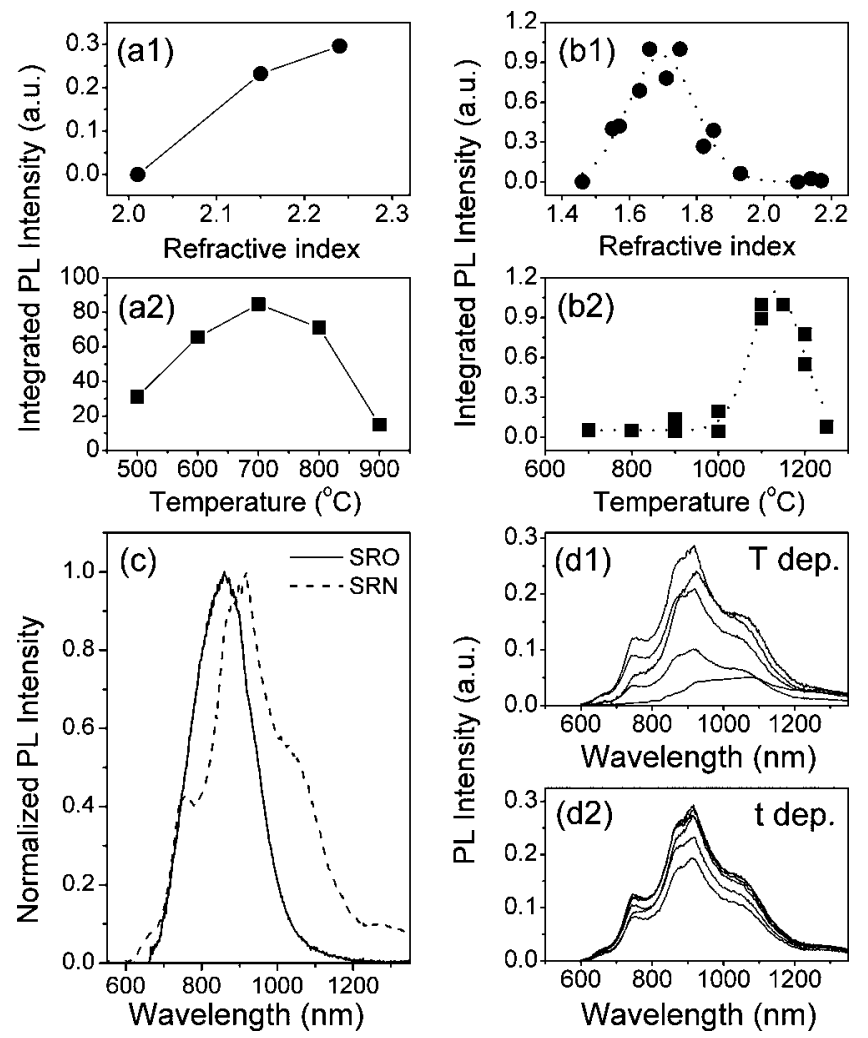

FIG. 1. (a1) Integrated PL intensity vs refractive index for SRN films annealed at $700{ }^{\circ} \mathrm{C}$ for $10 \mathrm{~min}$. (a2) Integrated PL intensity vs annealing temperature for SRN films with $n=2.24$ refractive index and annealed for 10 min. (b1) Integrated PL intensity vs refractive index for SRO films annealed at $1100{ }^{\circ} \mathrm{C}$ for $1 \mathrm{~h}$. (b2) Integrated PL intensity vs annealing temperature for SRO films with $n=1.7$ refractive index and annealed for $1 \mathrm{~h}$. (c) Normalized PL spectra of the optimized $(n=1.7$, annealed for $60 \mathrm{~min}$ at $\left.1100{ }^{\circ} \mathrm{C}\right)$ SRO film (solid line) and the best emitting $(n=2.24$, annealed for $10 \mathrm{~min}$ at $700^{\circ} \mathrm{C}$ ) SRN film (dash line). Pump power of $5 \mathrm{~mW}$ at $488 \mathrm{~nm}$. The oscillations in the SRN emission spectrum are due to interference induced from finite sample thickness $(700 \mathrm{~nm})$. (d1) PL spectra of SRN films vs annealing temperatures for a fixed annealing time of $10 \mathrm{~min}$. The sequence of PL intensities follows the trend of panel (a2) with the highest intensity at $700{ }^{\circ} \mathrm{C}$. (d2) PL spectra of SRN films vs annealing time for a fixed annealing temperature of $700{ }^{\circ} \mathrm{C}$. The annealing times are $1,2,5,10$, 20, and $60 \mathrm{~min}$ and the PL spectra show increasing peak intensity accordingly.

cross section transmission electron microscopy (TEM) analysis (Fig. 3, inset). Since the SRO integrated intensity decreases by increasing the SRO refractive index beyond a characteristic value $(\sim 1.7$ for our choice of deposition parameters) as shown in Fig. 1(b1), we refer to this situation as supersaturation limited. This trend was not observed in the case of the SRN films, where within the set of deposition conditions that we investigated, the integrated emission monotonically increases by increasing the film refractive index [Fig. 1(a1)].

In order to compare quantitatively the emission efficiency of SRO versus SRN light-emitting systems, we have measured the PLQE of the best emitting SRN film $\left(n=2.24,700{ }^{\circ} \mathrm{C}, 10 \mathrm{~min}\right)$ and the PLQE of the SRO sample with optimized emission characteristics $\left(n=1.7,1150{ }^{\circ} \mathrm{C}\right.$, $1 \mathrm{~h})$.

Figure 1(c) shows the normalized and almost overlapping room temperature emission spectra of the SRO (solid line) and SRN (dash line) samples that we compared. By direct PLQE measurements, performed as described in Ref. 20 , we have obtained a value of $7 \%$ for the SRN film versus

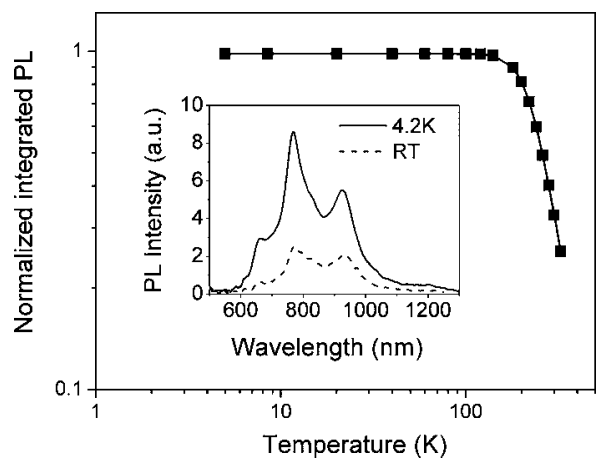

FIG. 2. (Squares) Integrated PL intensity vs temperature for the best emitting SRN sample $\left(n=2.24\right.$, annealed for $10 \mathrm{~min}$ at $\left.700^{\circ} \mathrm{C}\right)$. (Solid line) Theoretical fitting curve according to the model of Ref. 26 for the best fit we have used $E_{A}=117 \mathrm{meV}$ and $\beta=184$. (Inset) Room temperature (dash line) and $4.2 \mathrm{~K}$ (solid line) SRN emission spectra. The oscillations in the SRN emission spectra are due to interference induced from finite sample thickness $(700 \mathrm{~nm})$

the $4.5 \%$ PLQE of the optimized SRO sample. We notice that these efficiency results are particularly interesting since we have compared the efficiency of a fully optimized (in terms of stoichiometry and annealing temperature) SRO film, as shown in Figs. 1(b1) and 1(b2), with the best emitting SRN sample whose stoichiometry (excess Si content) does not show a fully optimized trend [Fig. 1(a1)].

Another important feature of light-emitting SRN films is that both the emission wavelength and emission line shape are almost independent of the annealing temperature and annealing time, as shown in Figs. 1(d1) and 1(d2), respectively. A very weak peak shift was also observed by changing the SRN stoichiometry in the range from 2.01 to 2.24 (not shown here), while the emission of the stoichiometric silicon nitride $(n=2.01)$ is too weak to be detected. The lack of emission tunability observed in our SRN systems strongly indicates that the origin of light emission is not determined by size dependent quantum confinement effects.

In order to gain more insight on the light-emitting mechanism of our Si-nc systems, we have performed temperature dependent and time resolved PL measurements. In Fig. 2 we show the integrated PL of SRN for temperatures ranging from 4 to $330 \mathrm{~K}$. We found that the SRN emission shows negligible temperature quenching (approximately a factor of 4) over the investigated temperature range. In addition, no appreciable emission line shape modifications have been observed from $4 \mathrm{~K}$ to room temperature (Fig. 2, inset). We notice that the temperature dependence of the PL that we have experimentally measured in SRN systems is different from the temperature behavior that is generally observed for Si-nc embedded in $\mathrm{SiO}_{2}$ matrices ${ }^{21}$ or for porous $\mathrm{Si}^{22}$ In oxygen passivated Si-nc systems, the PL temperature behavior has been accurately described within a model introduced by Calcott, ${ }^{23}$ that includes the splitting of the singlet-triplet excitonic states due to the exchange interaction in strongly confined silicon systems. It has been found that the trend of the Si-nc PL versus temperature follows a bell-shape behavior typically peaked around $100 \mathrm{~K}^{21}$ On the contrary, PL data shown in Fig. 2 can be accurately described by a simple phenomenological model based on thermal ionization from localized radiative traps, ${ }^{24}$ as similarly reported in Ref. 25. According to this model, the temperature dependent PL intensity at a given temperature $I_{\mathrm{PL}}(T)$ can be expressed as 


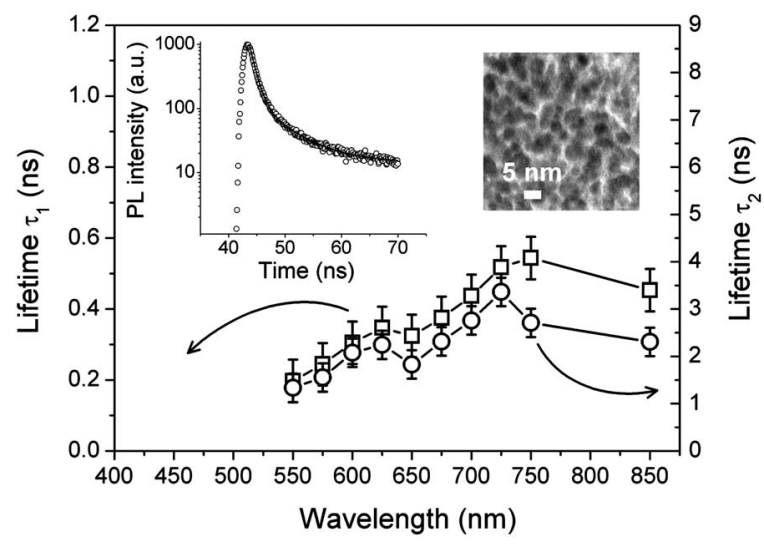

FIG. 3. PL emission lifetime vs observation wavelength for the best emitting SRN sample $\left(n=2.24\right.$, annealed for $10 \mathrm{~min}$ at $\left.700^{\circ} \mathrm{C}\right)$. A typical decay curve (observed at $710 \mathrm{~nm}$ ) is shown (left inset, open circles) together with a double exponential fit (left inset, solid line). Cross section TEM image of the best emitting SRN sample showing silicon nanocrystals as dark spots (right inset).

$I_{\mathrm{PL}}(T)=I(0)\left\{1 /\left[1+\beta \exp \left(-E_{A} / k_{B} T\right)\right]\right\}$ where $I(0)$ is the $\mathrm{PL}$ intensity at the lowest measured temperature, $\beta$ is a constant inversely proportional to the radiative rate of the system, ${ }^{24}$ and $E_{A}$ is an activation barrier which surrounds the recombination center. Both $\beta$ and $E_{A}$ are phenomenological constants whose microscopic origin is associated with the detailed nature of the interface region between the amorphous Si nitride matrix and the Si clusters. This model describes our data accurately with $E_{A}=117 \mathrm{meV}$ and $\beta=184$, in agreement with the results obtained in Ref. 25. However, here we propose a different interpretation of the nature of the radiative states involved in the photoluminescence process. Based on firstprinciples calculations of the optical properties of small size silicon clusters $(1-2 \mathrm{~nm})$ passivated with nitrogen, we have recently demonstrated that localized nitrogen states at the surface of Si-nc result in nanosecond relaxation times and large absorption/emission Stokes shifts. ${ }^{26}$ Therefore, the radiative nitrogen trap states responsible for light emission consists of localized states introduced within the optical gap of small Si clusters by bridging nitrogen configurations at the Si-nc surface. ${ }^{26}$

In Fig. 3 we show the measured wavelength dependent PL lifetime of the best emitting SRN film. We found that the SRN lifetime can be described by a double exponential function with a resolution-limited subnanosecond-fast decay component and a longer decay component that ranges between 1 and $5 \mathrm{~ns}$, depending on the observation wavelength. According to our interpretation of the light emission mechanism, the fast subnanosecond PL decay component is associated with the nonradiative exciton trapping time on the radiative nitrogen states while the longer (nanosecond) decay results from the recombination (both radiative and nonradiative) of the trapped excitons.

In addition, we observe that, because of the lack of emission tunability in our SRN system [Figs. 1(d1) and 1(d2)], the wavelength dispersion of the lifetime data shown in Fig. 3 cannot result from nanocrystal size dispersion. On the contrary, it is associated with the various types of $\mathrm{Si}-\mathrm{N}$ bonding groups passivating the interface between the Si clusters and the surrounding matrix. ${ }^{26}$

In conclusion, we have measured the PLQE of SRN and optimized SRO films fabricated by PECVD and magnetron sputtering, respectively, and we have demonstrated $7 \%$ PLQE for SRN samples. In addition we have demonstrated small emission temperature quenching from 4 to $330 \mathrm{~K}$ and nanosecond-fast, wavelength dependent recombination dynamics. The lack of emission tunability, the PL temperature behavior, and its emission dynamics suggest that the light emission originates from nitrogen-related surface states in small Si-ncs.

This work was supported by the MRSEC program of the National Science Foundation under Contract No. DMR 0213282. The authors thank S. Musikhin and A. Major for their help in PL lifetime measurement. The authors thank G. Galli, A. Williamson and S. Hamel for insightful discussions. The authors acknowledge Dr. X. Duan for TEM sample preparation and imaging. This work was partly supported by the Natural Science and Engineering Research Council of Canada under its Nano Innovations Platform, the Canada Research Chairs Programme, and the Canada Foundation for Innovation.

${ }^{1}$ L. T. Canham, Appl. Phys. Lett. 57, 1046 (1990).

${ }^{2}$ Z. H. Lu, D. J. Lockwood, and J. M. Baribeau, Nature (London) 378, 258 (1995).

${ }^{3}$ K. D. Hirschman, L. Tsybeskov, S. P. Duttagupta, and P. M. Fauchet, Nature (London) 384, 338 (1996).

${ }^{4}$ Silicon Photonics, edited by L. Pavesi and D. J. Lockwood (Springer, Berlin, 2004).

${ }^{5}$ J. C. Vial, A. Bsiesy, F. Gaspard, R. Hèrino, M. Ligeon, F. Muller, R. Romestain, and R. M. Macfarlane, Phys. Rev. B 45, 14171 (1992).

${ }^{6}$ B. Gelloz, T. Nakagawa, and N. Koshida, Appl. Phys. Lett. 73, 2021 (1998).

${ }^{7}$ B. Gelloz, Appl. Surf. Sci. 108, 449 (1997).

${ }^{8}$ L. Pavesi, L. Dal Negro, C. Mazzoleni, G. Franzó, and F. Priolo, Nature (London) 408, 440 (2000).

${ }^{9}$ L. Khriachtchev, M. Rasanen, S. Novikov, and J. Sinkkonen, Appl. Phys. Lett. 79, 1249 (2001).

${ }^{10}$ L. Dal Negro, M. Cazzanelli, L. Pavesi, S. Ossicini, D. Pacifici, G. Franzó, F. Priolo, and F. Iacona, Appl. Phys. Lett. 82, 4636 (2003).

${ }^{11}$ J. Ruan, P. M. Fauchet, L. Dal Negro, M. Cazzanelli, and L. Pavesi, Appl. Phys. Lett. 83, 5479 (2003).

${ }^{12}$ M. J. Chen, J. L. Yen, J. Y. Li, J. F. Chang, S. C. Tsai, and C. S. Tsai, Appl. Phys. Lett. 84, 2163 (2004).

${ }^{13}$ M. V. Wolkin, J. Jorne, P. M. Fauchet, G. Allan, and C. Delerue, Phys. Rev. Lett. 82, 197 (1999).

${ }^{14}$ A. J. Williamson, J. C. Grossman, R. Q. Hood, A. Puzder, and G. Galli, Phys. Rev. Lett. 89, 196803 (2002).

${ }^{15}$ E. Degoli, G. Cantele, E. Luppi, R. Magri, D. Ninno, O. Bisi, and S. Ossicini, Phys. Rev. B 69, 155411 (2004).

${ }^{16}$ N. M. Park, C. J. Choi, T. Y. Seong, and S. J. Park, Phys. Rev. Lett. 86, 1355 (2001).

${ }^{17}$ L. Dal Negro, J. H. Yi, V. Nguyen, Y. Yi, J. Michel, and L. C. Kimerling, Appl. Phys. Lett. 86, 261905 (2005).

${ }^{18}$ K. S. Cho, N. M. Park, T. Y. Kim, K. H. Kim, G. Y. Sung, and J. H. Shin, Appl. Phys. Lett. 86, 071909 (2005).

${ }^{19}$ M. Stolfi, L. Dal Negro, J. Michel, X. Duan, J. Le Blanc, J. Haavisto, and L. C. Kimerling, Mater. Res. Soc. Symp. Proc. 832, F.11.8.1 (2005).

${ }^{20}$ T.-W. F. Chang, A. Maria, P. W. Cyr, V. Sukhovatkin, L. Levina, and E. H. Sargent, Synth. Met. 148, 257 (2005).

${ }^{21}$ M. L. Brongersma, P. G. Kik, A. Polman, K. S. Min, and Harry A. Atwater, Appl. Phys. Lett. 76, 351 (2000).

${ }^{22}$ A. G. Cullis, L. T. Canham, and P. D. J. Calcott, J. Appl. Phys. 82, 909 (1997).

${ }^{23}$ P. D. J. Calcott, K. J. Nash, L. T. Canham, K. J. Kane, and D. Brumhead, J. Phys.: Condens. Matter 5, L91 (1993).

${ }^{24}$ J. I. Pankove, Optical Processes in Semiconductors (Dover, New York, 1971).

${ }^{25}$ S. V. Deshpande, E. Gulari, S. W. Brown, and S. C. Rand, J. Appl. Phys. 77, 6534 (1995).

${ }^{26}$ L. Dal Negro, J. H. Yi, L. C. Kimerling, S. Hamel, A. Williamson, and G. Galli, Appl. Phys. Lett. 88, 183103 (2006). 